International Journal of Basic and Applied Sciences, 1 (2) (2012) 124-136

(C) Science Publishing Corporation

www.sciencepubco.com/index.php/IJBAS

\title{
Effects of Additional Food on Predator-Prey Systems with Monotonic and Non-Monotonic Functional Response: A Comparative Study
}

\author{
Banshidhar Sahoo \\ Department of Mathematics, Daharpur A.P.K.B Vidyabhaban \\ Paschim Medinipur, West Bengal, India. \\ Email:banshivu@gmail.com
}

\begin{abstract}
In this paper, we have proposed predator-prey models with monotonic and non-monotonic functional response with additional food to top-predator. Bifurcation analysis of the proposed models confirm the vital role of response function in the controllability of the predator-prey system dynamics with additional food. We observe that additional food is a very powerful tool for controlling dynamics of a predator-prey model with monotonic functional response but not very useful for the system with non-monotonic response function.
\end{abstract}

Keywords: Predator-prey, Monotonic and Non-monotonic functional response, Additional food, Bifurcation.

\section{Introduction}

In population dynamics, a functional response is the rate at which each predator captures prey. The simplest form of functional response was obtained by assuming that in the time available for searching, the total change in the prey density is proportional to the prey density. Hence, if $x(t)$ represents the prey concentration at time $t$, then the functional response is $a x(t)$, where $a>0$ is a constant. Such a response was used almost simultaneously by Lotka in 1925 for studying a hypothetical chemical reaction and by Volterra in 1926 for modeling a predator-prey interaction. However, the curve defined by the 
Lotka-Volterra response function is a straight line through the origin and it is unbounded. But, the more natural response functions should be nonlinear and bounded. In 1913, Michaelis and Menten proposed a nonlinear response function $f(x)=\frac{m x}{a+x}$, where $m>0$ denotes the maximal growth rate of the species and $a>0$ is the half-saturation constant. Holling [1] also used this function as one of the predator functional responses. It is now referred to as a Michaelis-Menten function or a Holling type-II function. Another response function is $f(x)=\frac{m x^{2}}{a+b x+x^{2}}$, which is known as sigmoidal response function. It's simplfied form $f(x)=\frac{m x^{2}}{a+x^{2}}$ is known as Holling type-III response function. There is experimental and observational evidence which indicates that this need not always be the case, for example, in the cases of "inhibition "in microbial dynamics and "group defense "in population dynamics( Freedman et al. [2]), Mischaikow et al. [3], Wolkowicz [4] ). So many researchers (Andrews [5], Boon and Landelout [6], Yang and Humphrey [7], Ruan and Xiao [8] etc.) investigaed that non-monotonic responses occur at the microbial level: when the nutrient concentration reaches a high level an inhibitory effect on the specific growth rate may occur. This is often seen when microorganisms are used for waste decomposition or for water purification. To model such an inhibitory model Andrews [5] suggested a function $f(x)=\frac{m x}{a+b x+x^{2}}$ called the Monod-Haldane function or Holling type-IV function (Taylor [9]) for low concentrations but includes the inhibitory effect at high concentrations. Sokol and Howell [10] proposed a simplified Monod-Haldane function or Holling type-IV function of the form $f(x)=\frac{m x}{a+x^{2}}$ and found that it fits their experimental data significantly better and is simpler since it involves only two parameters. The nature of monotonic and non-monotonic functional responses are shown in figure 1.

The consequences of providing a predator with additional food and the corresponding effects on the predator-prey dynamics and its utility in biological control have been the great topic of study for many scientists due to its eco-friendly nature. In recent years, many biologist, experimentalists, and theoreticians have investigated the consequences of providing additional food to predators in a predator-prey systems ( Bilde and Toft [11]), Coll and Guershon [12], Harmon [13], Harwood et al.[14], Murdoch et al.[15], Sabelis and van Rijn [16], Srinivasu et al. [17], Van Baalen et al. [18], Van Rijn et al. [19] ). Srinivasu et al. [17] investigated qualitative behavior of a predatorprey system in presence of additional food to the predator. They concluded that handling times for the available foods to the predator play the key role in determining the eventual state of the eco-system. Sahoo et al. [20] proposed a food chain model with seasonal effects on additional food. Sahoo [21] discussed the extinction criteria of species in a system depends on interaction functions and supply of the quality of additional food. A predator-prey system with 


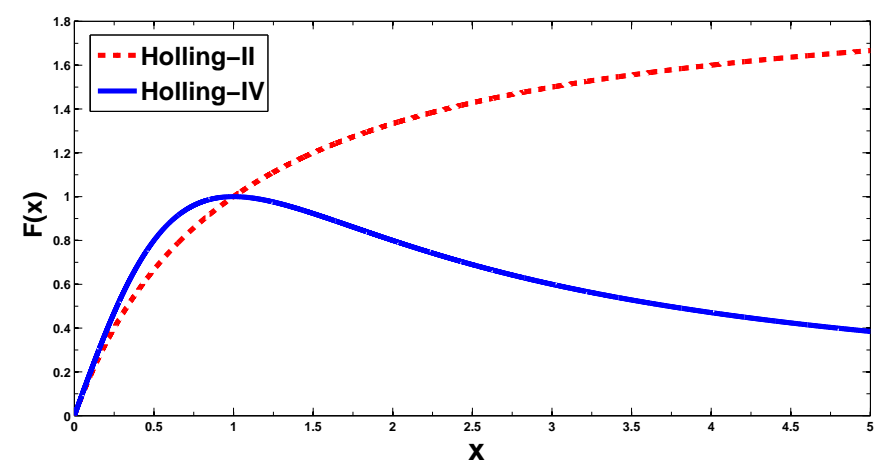

Figure 1: Red colour dash ( - ) curve represents the monotonic functional response $f(x)=\frac{m x}{a+x}$ and blue colour line curve represents the non-monotonic functional response $f(x)=\frac{m x}{a+x^{2}}$ with $m=2, a=1$.

different growth rates and different functional response is analysed and the results of the system with additional food is compared by Sahoo [22]. In very recent, Sahoo [23] proposed a consumer-nutrient-predator model and observed that for biological conservation, additional food plays a vital role for servival of consumer species in an ecosystem.

Srinivasu et al. [17] have investigated the effects of additional food to predators in a di-trophic food chain model. But, a tri-trophic food chain model (Hastings and Powell [24]) is much more realistic than any di-trophic food chain model. Therefore the study of tri-trophic food chain model with additional food is necessary. In this paper, we have investigated the effects of additional food (characterized by predator's handling time) to top-predator in tri-trophic food chain model with monotonic as well as non-monotonic functional responses. The main object of this paper is to compair the effects of additional food with monotonic and non-monotonic functional responses on the controllability of predator-prey dynamics. Bifurcation analysis is done with respect to quality and quantity of additional food and with respect to saturation constant.

\section{Models}

The famous HP [24] model with pairwise interactions between three species, namely, $X, Y, Z$, which incorporates a Holling type-II functional interactions in both consumer species is the following 


$$
\begin{aligned}
\frac{d X}{d T} & =R_{0} X\left(1-\frac{X}{K_{0}}\right)-C_{1} A_{1} \frac{X Y}{B_{1}+X} \\
\frac{d Y}{d T} & =A_{1} \frac{X Y}{B_{1}+X}-A_{2} \frac{Y Z}{B_{2}+Y}-D_{1} Y \\
\frac{d Z}{d T} & =C_{2} A_{2} \frac{Y Z}{B_{2}+Y}-D_{2} Z
\end{aligned}
$$

Now, we consider a tri-trophic food chain model with Monod-Haldane response function ( non-monotonic functional response ) of the form

$$
\begin{aligned}
& \frac{d X}{d T}=R_{0} X\left(1-\frac{X}{K_{0}}\right)-C_{1} A_{1} \frac{X Y}{B_{1}+X^{2}} \\
& \frac{d Y}{d T}=A_{1} \frac{X Y}{B_{1}+X^{2}}-A_{2} \frac{Y Z}{B_{2}+Y^{2}}-D_{1} Y \\
& \frac{d Z}{d T}=C_{2} A_{2} \frac{Y Z}{B_{2}+Y^{2}}-D_{2} Z
\end{aligned}
$$

Here $X$ be the numbers of species at lowest level of the food chain, $Y$ the numbers of the species that preys upon $X$ and $Z$ the numbers of the species that preys upon $Y$. Here $T$ is time. The constant $R_{0}$ is the "intrinsic growth rate" and the constant $K_{0}$ is the "carrying capacity" of the species $X$. The constant $C_{1}^{-1}$ and $C_{2}$ are conversion rates of prey to predators for species $Y$ and $Z$ respectively; $D_{1}$ and $D_{2}$ are constant death rates for species $Y$ and $Z$ respectively. The constants $A_{i}$ and $B_{i}$ for $i=1,2$ are maximal predation rate and half saturation constants for $Y$ and $Z$ respectively.

If $h_{1}$ and $e_{1}$ are constants representing handling time of the top-predator $Z$ per intermediate item and ability of the predators to detect the prey then we have $A_{2}$ and $B_{2}$, representing the maximum predation rate and half saturation values of the top-predator $Z$, to be $1 / h_{1}$ and $1 / e_{1} h_{1}$, respectively.

Now, we modify the model (1) and model (2) by providing "additional food"to predators population. We make the following assumptions:

(a) The prey population grows as per logistic equation in the absense of predators.

(b) Predators are provided with additional food of constant biomass $A$ which is distributed uniformly in the habitat.

(c) The number of encounters per predator with the additional food is proportional to the density of the additional food.

(d) The proportionality constant characterizes the ability of the predator to 
identify the additional food.

With the above assumptions, the Hastings and Powell model (1) takes the following form:

$$
\begin{aligned}
\frac{d X}{d T} & =R_{0} X\left(1-\frac{X}{K_{0}}\right)-C_{1} A_{1} \frac{X Y}{B_{1}+X} \\
\frac{d Y}{d T} & =A_{1} \frac{X Y}{B_{1}+X}-A_{2} \frac{Y Z}{B_{2}+\alpha \mu A+Y}-D_{1} Y \\
\frac{d Z}{d T} & =C_{2} A_{2} \frac{(Y+\mu A) Z}{B_{2}+\alpha \mu A+Y}-D_{2} Z
\end{aligned}
$$

and the model (2) takes the form:

$$
\begin{aligned}
\frac{d X}{d T} & =R_{0} X\left(1-\frac{X}{K_{0}}\right)-C_{1} A_{1} \frac{X Y}{B_{1}+X^{2}} \\
\frac{d Y}{d T} & =A_{1} \frac{X Y}{B_{1}+X^{2}}-A_{2} \frac{Y Z}{B_{2}+\alpha \mu A+Y^{2}}-D_{1} Y \\
\frac{d Z}{d T} & =C_{2} A_{2} \frac{(Y+\mu A) Z}{B_{2}+\alpha \mu A+Y^{2}}-D_{2} Z
\end{aligned}
$$

If $h_{2}$ represents the handaling time of the top-predator $Z$ per unit quantity of additional food and $e_{2}$ represents the ability for the top-predator $Z$ to detect the additional food, then we have $\mu=e_{2} / e_{1}$ and $\alpha=h_{2} / h_{1}$. The terms $\mu A$ represents effectual additional food for the top-predator $Z$.

We nondimensionalize the model (3) and (4) with $x=\frac{X}{K_{0}}, y=\frac{C_{1} Y}{K_{0}}$, $z=\frac{C_{1} Z}{C_{2} K_{0}}, t=R_{0} T$ and we obtain the following systems

$$
\begin{aligned}
\frac{d x}{d t} & =x(1-x)-\frac{a_{1} x y}{1+b_{1} x} \\
\frac{d y}{d t} & =\frac{a_{1} x y}{1+b_{1} x}-\frac{a_{2} y z}{1+\alpha \xi+b_{2} y}-d_{1} y \\
\frac{d z}{d t} & =\frac{\beta(y+c \xi) z}{1+\alpha \xi+b_{2} y}-d_{2} z
\end{aligned}
$$

and

$$
\begin{aligned}
\frac{d x}{d t} & =x(1-x)-\frac{a_{1} x y}{1+b_{1} x^{2}} \\
\frac{d y}{d t} & =\frac{a_{1} x y}{1+b_{1} x^{2}}-\frac{a_{2} y z}{1+\alpha \xi+b_{2} y^{2}}-d_{1} y \\
\frac{d z}{d t} & =\frac{\beta(y+c \xi) z}{1+\alpha \xi+b_{2} y^{2}}-d_{2} z
\end{aligned}
$$


where

$a_{1}=\frac{A_{1} K_{0}}{R_{0} B_{1}}, a_{2}=\frac{A_{2} K_{0} C_{2}}{B_{2} R_{0} C_{1}}, b_{1}=\frac{K_{0}}{B_{1}}, b_{2}=\frac{K_{0}}{B_{2} C_{1}}, c=\frac{C_{1} B_{2}}{K_{0}}, \xi=\frac{\mu A}{B_{2}}, d_{1}=\frac{D_{1}}{R_{0}}$, $d_{2}=\frac{D_{2}}{R_{0}}$. The systems (5) and (6) are to be analyzed with the following initial conditions: $x(0)>0, y(0)>0, z(0)>0$.

Here $a_{1}$ and $a_{2}$ represent prey and intermediate predator consumption rate, $b_{1}$ and $b_{2}$ represent prey and intermediate predator saturation constant, $d_{1}$ and $d_{2}$ represent intermediate predator death rate and top-predator death rate, $\beta$ represents conversion rate of intermediate predator to top-predator, $c$ is the conversion rate of quantity of additional food. The parameter $\alpha$ represents the "quality" of the additional food ( ratio between top-predator's handling time towards additional food and per intermediate predator item) and $\xi$ represents the "quantity" of the additional food for top-predator. Here $\alpha, \xi$ are the paramaters which characterize the additional food for top-predator. Now, we shall analyze the dynamics of the systems (5) and (6) and compare the effects of monotonic and non-monotonic functional response on the dynamics.

\section{Results}

We have done the bifurcation analysis of the systems (5) and (6) with respect to the parameter $b_{1}$, quality of additional food $\alpha$ and quantity of additional food $\xi$ respectively, with the set of ecosystem parameters values $a_{1}=5.0$, $a_{2}=0.1, b_{2}=2.0, c=0.85, \beta=0.08, d_{1}=0.4, d_{2}=0.01$, which are taken from Hastings and Powell model [24]. The remaining three parameters $b_{1}, \alpha$ (quality of additional food) and $\xi$ (quantity of additional food) are varied to perform the bifurcation analysis of the systems.

\subsection{Bifurcation analysis with respect to $b_{1}$}

Figure 2 is the bifurcation analysis of system (5) and (6) with respect to the parameter $b_{1}$ with out supply of additional food (i.e, for $\alpha=0, \xi=0$ ). From figure 2(a), we observe that system (5) has stable coexistence for $1.4<b_{1}<2.2$. After $b_{1} \geq 2.2$, the system (5) depicts chaotic behaviour. The system (6) has stable coexistence for $1.4<b_{1}<1.9$, chaotic attractors for $1.9 \leq b_{1}<3.4$ and for $3.4 \leq b_{1} \leq 7$, it has limit cycle oscillation which is observed in figure $2(\mathrm{~b})$.

Figure 3 is the bifurcation analysis of system (5) and (6) with respect to the parameter $b_{1}$ with additional food $\alpha=2, \xi=0.04$. From figure 3(a), we observe that system (5) is reported stable coexistence for $1<b_{1}<1.7$, limit cycle behaviour for $1.7 \leq b_{1}<2.62$. After $b_{1} \geq 2.62$, the system (5) shows high periodic and chaotic behaviour. From figure $3(\mathrm{~b})$, we observe that the system (6) has stable coexistence for $0.8<b_{1} \leq 1.35$, periodic behaviour for 


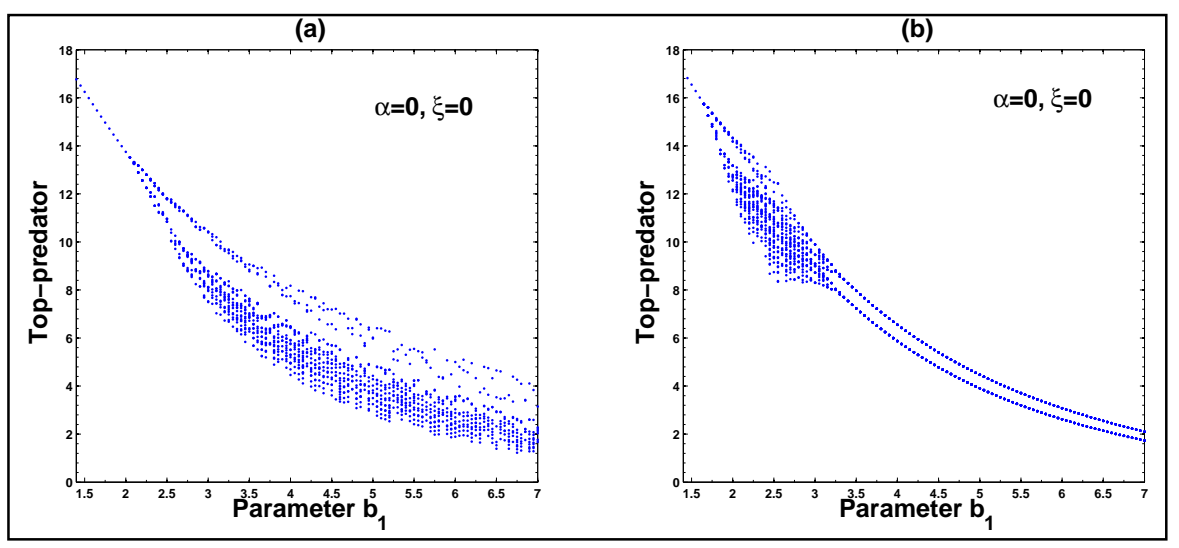

Figure 2: Bifurcation diagram with respect to the parameter $b_{1}$ with out supply of additional food $(\alpha=0, \xi=0)$. Diagrams (a) is for the system (5) and (b) is for the system $(6)$.

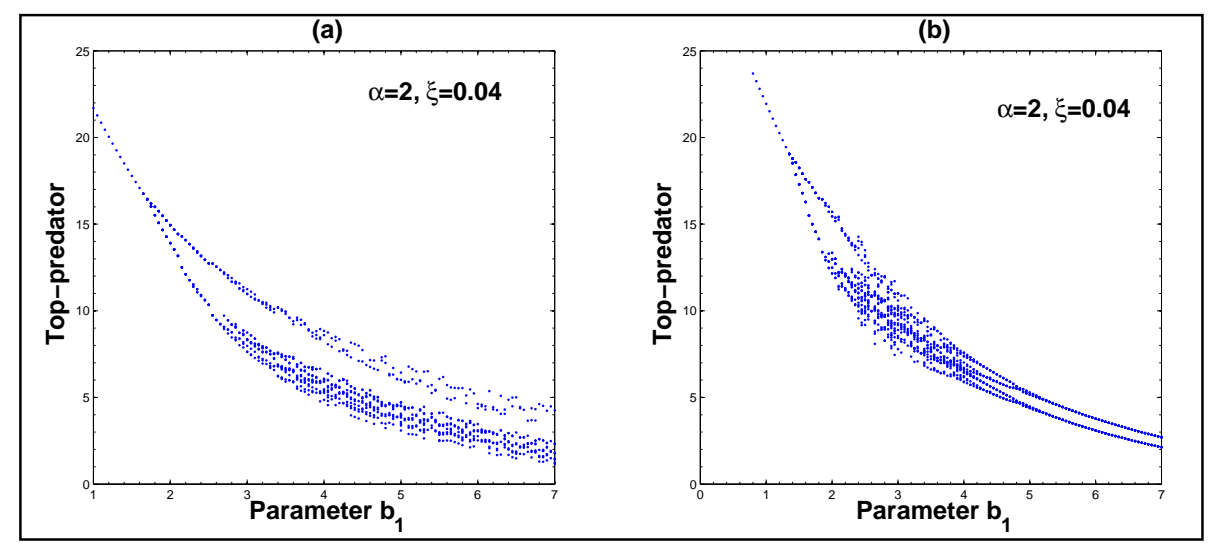

Figure 3: Bifurcation diagram with respect to the parameter $b_{1}$ with additional food $\alpha=2, \xi=0.04$. Diagrams (a) is for the system (5) and (b) is for the system (6). 


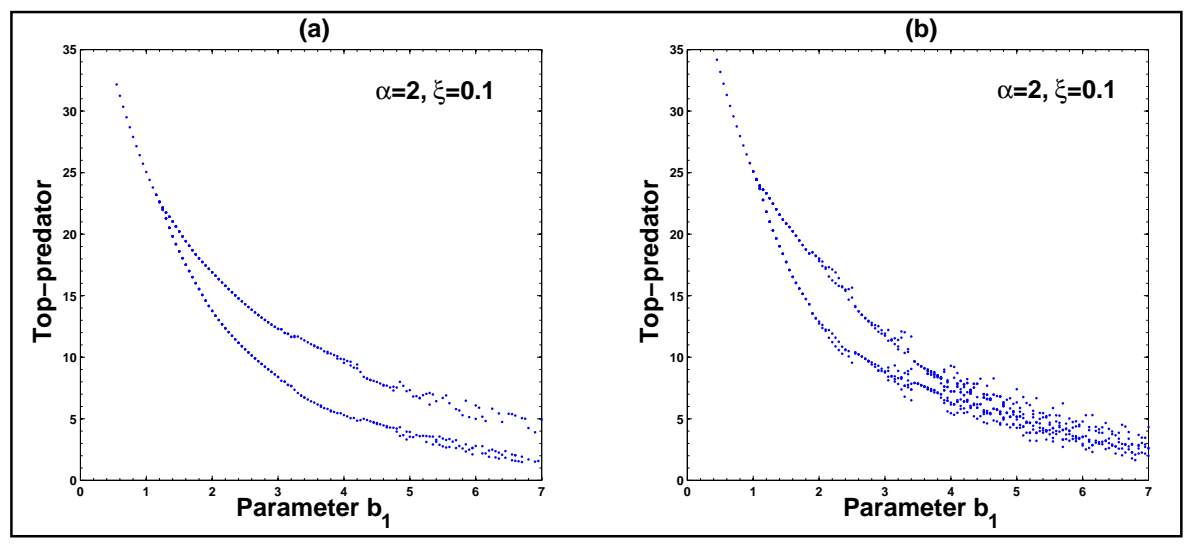

Figure 4: Bifurcation diagram with respect to the parameter $b_{1}$ with additional food $\alpha=2, \xi=0.1$. Diagrams (a) is for the system (5) and (b) is for the system (6).

$1.35<b_{1}<1.9$, chaotic attractors and high periodicity for $1.9 \leq b_{1}<5.2$ and for $5.2 \leq b_{1} \leq 7$, it has limit cycle oscillation.

The bifurcation analysis of the systems (5) and (6) are done with respect to the parameter $b_{1}$ for $\alpha=2, \xi=0.1$ which is shown in figure 4 . From figure 4(a) we observe that the system (5) is predicted stable steady state for $0.81<b_{1}<1.2$ and periodic orbits for $1.2 \leq b_{1}<7$. Therefore the chaotic behaviour vanishes from the system (5) for supply of this type of additional food. From figure 4(b), it is observe that the system (6) have steady state behaviour for $0.65<b_{1}<1$. , limit cycle behaviour for $1.1<b_{1} \leq 2$, and high periodic and chaotic behaviour for $2<b_{1} \leq 7$.

\subsection{Bifurcation analysis with respect to quality of addi- tional food $\alpha$}

Figure 5 is the bifurcation diagram of the system (5) and (6) with respect to quality of additional food $\alpha$ fixing $\xi=0.1, b_{1}=3$. Figure 5 (a) shows that the system (5) has limit cycle behaviour for $0 \leq \alpha<8.9$ and it settles down to steady state for $8.9 \leq \alpha \leq 10$. From figure $5(\mathrm{~b})$, we observe periodic and chaotic attractors for differet ranges of $0 \leq \alpha \leq 10$. Therefore, the system (5) depicts periodic behaviour and the system (6) shows chaotic behaviour with respect to quality of additional food $\alpha$. 


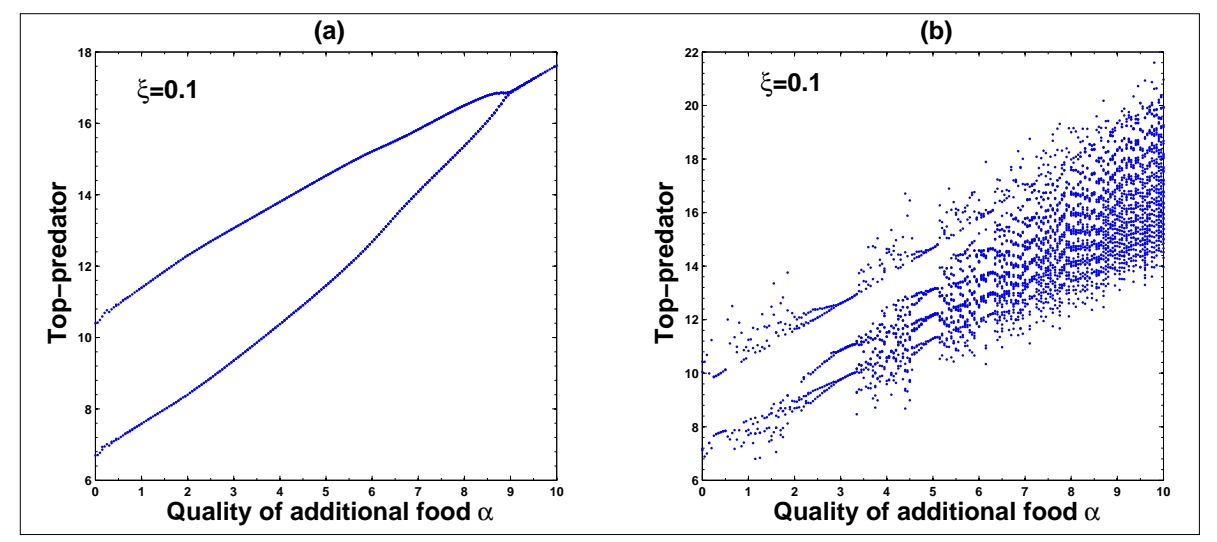

Figure 5: Bifurcation diagram with respect to quality of additional food $\alpha$ for $\xi=0.1, b_{1}=3$. Diagrams (a) is for the system (5) and (b) is for the system $(6)$.

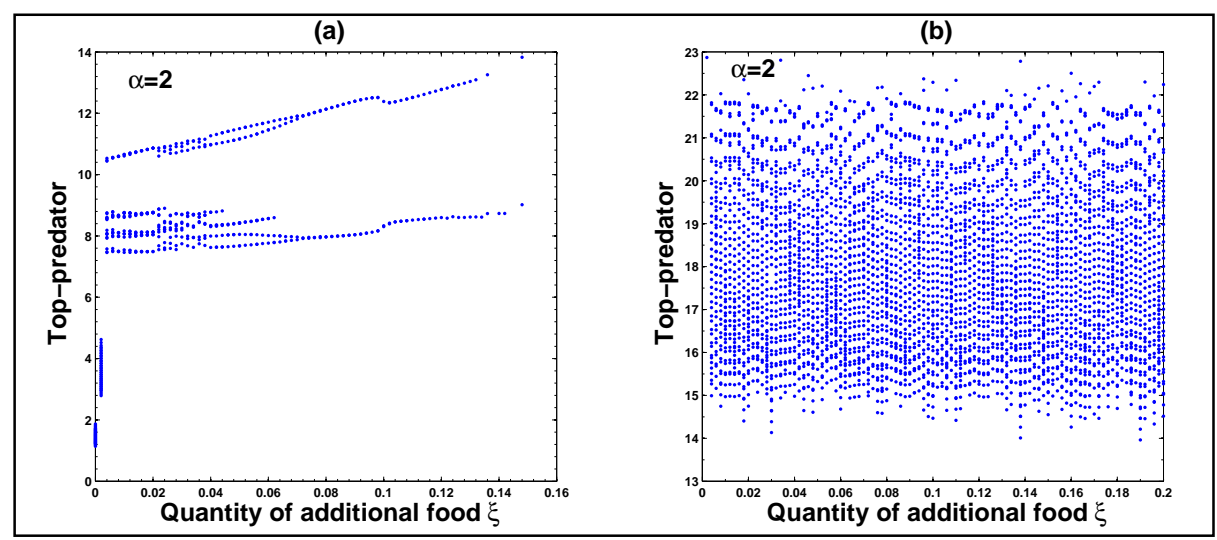

Figure 6: Bifurcation diagram with respect to quantity of additional food $\xi$ for $\alpha=2, b_{1}=3$. Diagrams (a) is for the system (5) and (b) is for the system (6). 


\subsection{Bifurcation analysis with respect to quantity of addi- tional food $\xi$}

Figure 6 is the bifurcation analysis of the system (5) and (6) with respect to quantity of additional food $\xi$ fixing $\alpha=2, b_{1}=3$. From figure $6(\mathrm{a})$, we observe periodic behaviour for $0 \leq \xi<1.55$. But the system (6) is reported chaotic behaviour for $0 \leq \xi \leq 0.2$ which is shown in figure $6(\mathrm{~b})$.

The dynamical behaviors of the system (5) and the system (6) are shown in tabular form in table 1 and table 2 respectively.

\begin{tabular}{|c|l|l|}
\hline Bifurcation parameters & Fixed parameter values & Observations \\
\hline$b_{1}$ & $\alpha=0, \xi=0$ & Stability, periodicity, chaos \\
$b_{1}$ & $\alpha=2, \xi=0.04$ & Stability, periodicity, chaos \\
$b_{1}$ & $\alpha=2, \xi=0.1$ & Stability, periodicity \\
$\alpha$ & $\xi=0.1, b_{1}=3$ & Limit cycle, stability \\
$\xi$ & $\alpha=2, b_{1}=3$ & Periodicity \\
\hline
\end{tabular}

Table 1: Behaviour of the system (5) with respect to the parameters $b_{1}, \alpha, \xi$.

\begin{tabular}{|c|l|l|}
\hline Bifurcation parameters & Fixed parameter values & Observations \\
\hline$b_{1}$ & $\alpha=0, \xi=0$ & Stability, periodicity, chaos \\
$b_{1}$ & $\alpha=2, \xi=0.04$ & Stability, periodicity, chaos \\
$b_{1}$ & $\alpha=2, \xi=0.1$ & Stability, periodicity, Chaos \\
$\alpha$ & $\xi=0.1, b_{1}=3$ & Periodicity, Chaos \\
$\xi$ & $\alpha=2, b_{1}=3$ & Chaos \\
\hline
\end{tabular}

Table 2: Behaviour of the system (6) with respect to the parameters $b_{1}, \alpha, \xi$.

\section{Discussion}

We have proposed three species predator-prey systems using monotonic (Holling type-II) and non-monotonic (Holling type-IV) functional response in presence of some additional food to top-predator. We have analysed the systems behaviour through bifurcation study with out additional food as well as with additional food. We mainly focus on the effects of additional food to the systems of monotonic and non-monotonic functional responses. Table 1 and table 2 are reported the dynamical behaviors of the system (5) and (6) respectively. With out any supply of additional food, both monotonic and 
non-monotonic systems have chaotic attractors. But, the chaos is controlled by supply of additional food in the monotonic system, while the non-monotonic system shows chaotic behavior through supply of additional food. Therefore, we conclude that for models with monotonic functional response with additional food makes very well to control the dynamics but it is not very useful for model with non-monotonic response function. Our analysis will be useful in fishery and biological pest control research for use of additional food with propoer choice of response function.

\section{Acknowledgements}

The author is very grateful to Dr. Swarup Poria for his valuable suggestions which have helped in a better exposition of the paper.

\section{References}

[1] C.S. Holling, The functional response of predators to prey density and its role in mimicry and population regulation, Mem Entomolog Soc Can. 97(45) (1965) 1-60.

[2] H.I. Freedman , G.S.K. Wolkowicz, Predator-prey systems with group defence: The paradox of enrichment revisited, Bull Math. Biol. 48 (1986) 493-508.

[3] K. Mischaikow , G.S.K. Wolkowicz, predator-prey system involving group defense: A connection matrix approach, Nonlinear Anal. 14 (1990) 955969.

[4] G.S.K. Wolkowicz, Bifurcation analysis of a predator-prey system involving group defence, SIAM J. Appl. Math. 48 (1988) 592-606.

[5] J.F. Andrews, A mathematical model for the continuous culture of microorganisms utilizing inhibitory substrates, Biotechnol. Bioeng. 10 (1968) 707-723.

[6] B. Boon, H. Landelout, Kinetics of nitrite oxidation by nitrobacter winogradski, Biochem. J., 85 (1962), 440-447.

[7] R. D. Yang, A. E. Humphrey, Dynamics and steady state studies of phenol biodegeneration in pure and mixed cultures, Biotechnol. Bioeng. 17 (1975) 1211-1235. 
[8] S.Ruan, D. Xiao, Global analysis in a predator-prey system with nonmonotonic functional response, Siam J. Appl. Math. 61 (2001) 1445-1472.

[9] R. J. Taylor, Predation, Chapman and Hall, New York, 1984.

[10] W. Sokol, J.A. Howell, Kinetics of phenol oxidation by washed cells, Biotechnol. Bioeng. 23 (1980) 2039-2049.

[11] T. Bilde, S. Toft, Quantifying food limitation of arthropod predators in the field, Oecologia 115 (1998) 54-58.

[12] M. Coll , M. Guershon, Omnivoryin terrestrial arthropods: mixing plant and prey diets, Annu Rev Entomol 47 (2002) 267-297.

[13] J.P. Harmon, Indirect interactions among a generalist predator and its multiple foods, Ph.D thesis, St Paul MN University of Minnesota, 2003.

[14] J.D. Harwood, J.J. Obrycki, The role of alternative prey in sustaining predator populations, In: HoddleMS (ed) Proc. second int. symp. biol. control of arthropods, vol II (2005b) 453-462.

[15] W.W. Murdoch, J. Chesson, P.L. Chesson, Biological control in theory and practice, Am Nat. 125(3) (1985) 344-366.

[16] M.W. Sabelis, P.C.J. van Rijn, When does alternative food promote biological pest control? In: Hoddle MS (ed) Proc. second int. symp. biol. control of arthropods, vol II (2005) 428-437.

[17] P.D.N. Srinivasu, B.S.R.V. Prasad, M. Venkatesulu, Biological control through provision of additional food to predators: a theoretical study, Theor. Popul. Biol. 72 (2007) 111-120.

[18] M. Van Baalen, V. Kivan, P.C.J. Van Rijn, M.W. Sabelis, Alternative food, switching predators, and the Persistence of PredatorPrey Systems, Am Nat. 157(5) (2001) 512-524.

[19] P.C.J. Van Rijn, Y.M. Van Houten, M.W. Sabelis, How plants benefit from providing food to predators even when it is also edible to herbivores, Ecology 83 (2002) 2664-2679.

[20] B. Sahoo, S. Poria, Dynamics of a Predator-prey System with Seasonal Effects on Additional Food, Int. J. Ecosy. 1 (2011) 10-13.

[21] B. Sahoo, A Predator-Prey Model with General Holling Interactions in Presence of Additional Food, Int. J. Plant Research. 2 (2012) 47-50. 
[22] B. Sahoo, Predator-prey Model with Different Growth Rates and Different Functional Responses: A Comparative Study with Additional Food, Int. J. Appl. Math. Research, 1 (2) (2012) 117-129.

[23] B. Sahoo, Effects of Additional Foods to Predators on NutrientConsumer-Predator Food Chain Model, ISRN Biomathematics, doi:10.5402/2012/796783.

[24] A. Hastings, T. Powell, Chaos in a three-species food chain, Ecology 72 (1991) 896-903. 